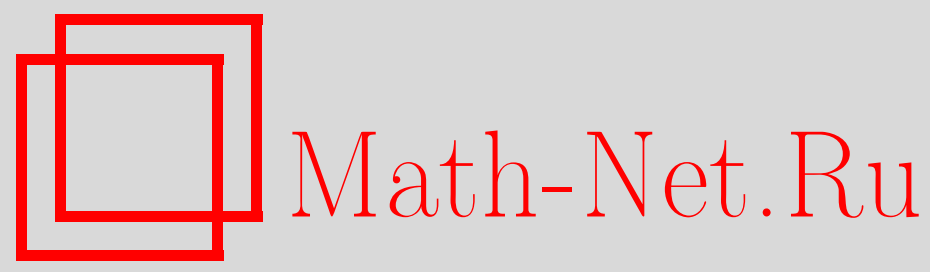

А. В. Гласко, Мера упорядоченности в статистических системах, Матем. заметки, 2003, том 74, выпуск 3, 350-361

DOI: https://doi.org/10.4213/mzm269

Использование Общероссийского математического портала Math-Net.Ru подразумевает, что вы прочитали и согласны с пользовательским соглашением http://www . mathnet.ru/rus/agreement

Параметры загрузки:

IP: 34.229 .108 .108

26 апреля 2023 г., 15:16:39

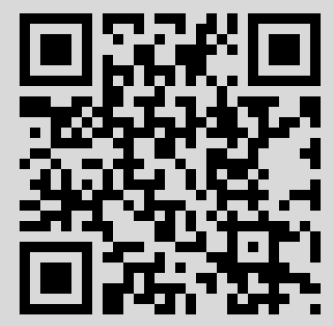




\title{
МЕРА УПОРЯДОЧЕННОСТИ В СТАТИСТИЧЕСКИХ СИСТЕМАХ
}

\author{
А. В. Гласко
}

Определяется феноменологическая мера упорядоченности в статистических системах. На основе этой меры строится формальная модель системы, развивающейся с ростом управляюшего параметра по закону наибыстрейшего роста порядка.

Библиография: 12 названий.

1. Введение. Известно [1]-[3], что некоторые физические системы обладают способностью переходить из неупорядоченного состояния в упорядоченное в результате изменения некоторого параметра. Классическим примером такой системы может служить ферромагнетик [4], [1]: при температурах

$$
T \geqslant T_{c}
$$

где $T_{c}$ - температура Кюри, спины различных атомов ферромагнетика ориентированы хаотически; по-мере же уменьшения температуры спины выстраиваются в одном (спонтанно выбранном) направлении и в пределе при $T / T_{c} \rightarrow 0$ образец превращается в монодомен. В настоящей работе развивается математическая схема описания процессов упорядочения (самоорганизации) в статистических системах. Основным математическим объектом этой схемы является мера упорядоченности в системе. В настоящеевремя известно несколько различных определений меры упорядоченности [5], [3], [6], [7]. Основываясь на двух физических моделях: фазового перехода 1-го рода (кристаллизация) и фазового перехода 2-го рода (возникновение спонтанной намагниченности), мы вводим новое, феноменологическое, определение меры упорядоченности. Эта мера позволяет построить формальную модель системы, развивающейся с ростом управляющего параметра по закону наибыстрейшего роста порядка.

2. Определение меры упорядоченности. Рассмотрим для простоты одномерную кристаллическую решетку с периодом $a$. При температурах больших абсолютного нуля $(T>0)$ решетка неидеальна: в ней присутствуют дефекты и фононы [8]. Будем описьвать состояние решетки плотностью вероятности $y(x)$ отклонения $x$ случайно выбранного атома от соответствующего ему узла. При $T=0$ решетка идеальна (в рамках классического описания) и каждый атом находится в соответствующем ему узле: $y(x)=\delta(x)$, где $\delta(x)-\delta$-функция Дирака. Это наиболее упорядоченное состояние решетки. Напротив, в точке плавления $\left(T=T_{0}\right)$ кристаллическая структура полностью отсутствует и атом с одинаковой вероятностью можно обнаружить в любой точке из 
интервала $x \in(-a, a): y(x)=y_{0}(x) \equiv 1 / 2 a$. Это состояние решетки наиболее хаотично.

Обозначим через $\mathscr{O}(y)$ меру упорядоченности в состоянии $y$ и шкалируем ее так, что

$$
\mathscr{O}\left(y_{0}\right)=0, \quad \mathscr{O}(\delta)=1
$$

При температурах $T \in\left(0, T_{0}\right)$ естественно определить меру упорядоченности как меру близости состояния системы к наиболее упорядоченному состоянию:

$$
\mathscr{O}(y)=1-C \rho^{2}(y, \delta)
$$

где $\rho$ - метрика на $\{y\}, C$ - нормировочный множитель. Правая часть в (2) построена так, что автоматически выполняется второе условие в (1). Множитель $C$ определим из первого условия:

$$
C=\frac{1}{\rho^{2}\left(y_{0}, \delta\right)} \text {. }
$$

Определение (2) нетрудно обобщить на другие системы. Рассмотрим, например, модель изотропного ферромагнетика [4], [1]. Каждьй атом охарактеризуем классическим спином (магнитньм моментом) s. В точке Кюри $\left(T=T_{c}\right)$ спины различных атомов ориентированы хаотически. При $T \rightarrow 0$ спины выстраиваются в одном направлении, которое мы примем за направление оси $z$. Будем описьвать состояние системы спинов плотностью вероятности $y(\theta)$ ориентации случайно выбранного спина под углом $\theta$ к оси $z$. В этом случае наиболее хаотичному состоянию (при $T=T_{c}$ ) соответствует равномерное распределение: $y(\theta)=1 / 2 \pi$, а наиболее упорядоченному (при $T=0)-\delta$-функция: $y(\theta)=\delta(\theta)$. Меру упорядоченности в промежуточных состояниях (при $\left.T \in\left(0, T_{c}\right)\right)$ определим формулой $(2)$.

В общем случае меру упорядоченности (2) будем рассматривать как характеристику плотности вероятности $y(x)$ некоторой непрерьвно распределенной на интервале $(-a, a)$ случайной величины $x$.

Перейдем теперь к конкретизации метрики $\rho$. Если бы $\delta$-функция была функцией в обычном смысле, то в качестве $\rho$ было бы естественно использовать гильбертову метрику. Однако поскольку $\delta(x)$ - обобщенная функция, этот выбор сразу приводит к расходимостям (по причине нелинейности гильбертовой метрики). С другой стороны, плотность $y(x)$ можно рассматривать [9] как регулярную обобщенную функцию, определенную на множестве основных функций $\Phi=\Phi(-a, a)$ :

$$
(y, \varphi)=\int_{-a}^{a} y(x) \varphi(x) d x, \quad \varphi \in \Phi .
$$

При этом в качестве $\rho$ можно взять гильбертову метрику на множестве обобщенных функций. Обобщенная функция - есть линейный непрерьвньй функционал, поэтому в качестве последней следует использовать гильбертову метрику на множестве функционалов, т.е. так называемую метрику множества функций с интегрируемым квадратом $L_{2}[10]$ :

$$
\rho\left(f_{1}, f_{2}\right)=\sqrt{\int_{\Phi}\left(f_{1}(\varphi)-f_{2}(\varphi)\right)^{2} d \mu}, \quad f_{1}, f_{2} \in L_{2} .
$$


Эта метрика отличается от обычной гильбертовой тем, что фигурирующий в ее определении интеграл есть интеграл Лебега по некоторой мере $\mu$ на множестве $\Phi$. В нашем случае

$$
f_{1}(\varphi)=(y, \varphi), \quad f_{2}(\varphi)=(\delta, \varphi)=\varphi(0),
$$

поэтому

$$
\rho(y, \delta)=\sqrt{\int_{\Phi}((y, \varphi)-\varphi(0))^{2} d \mu} .
$$

Итак окончательно определим меру упорядоченности в виде

$$
\mathscr{O}(y)=1-C \int_{\Phi}\left(\int_{-a}^{a} y(x) \varphi(x) d x-\varphi(0)\right)^{2} d \mu
$$

Меру $\mu$ выберем так, чтобы она обращалась в нуль только на конечных или счетных множествах (смысл этого требования будет ясен при исследовании свойств меры упорядоченности в разделе 3$)$. Множитель $C$ в соответствии с (3) равен

$$
C=\left[\int_{\Phi}\left(\frac{1}{2 a} \int_{-a}^{a} \varphi(x) d x-\varphi(0)\right)^{2} d \mu\right]^{-1}=\left[\int_{\Phi}\left(\langle\varphi(x)\rangle_{y_{0}}-\varphi(0)\right)^{2} d \mu\right]^{-1}
$$

где $\langle\varphi\rangle_{y_{0}}$ - среднее значение функции $\varphi(x)$ по равномерному распределению $y_{0}$.

Отметим, что определение (7) неоднозначно: конкретная мера упорядоченности зависит от выбора меры множества основных функций, т.е. $\mathscr{O}(y)=\mathscr{O}_{\mu}(y)$.

3. Свойства меры упорядоченности. Свойства меры упорядоченности в основном определяются общим аксиоматическим определением метрики и свойствами метрики (5).

ЛЕмма 1. Мера упорядоченности в системе, характеризуемой равномерным распределением равна нулю: $\mathscr{O}\left(y_{0}\right)=0$.

Это свойство очевидно из (7), (8).

ЛЕмма $2 . \mathscr{O}(y) \leqslant 1$, причем $\mathscr{O}(y)=1 \Longleftrightarrow y(x)=\delta(x)$.

ДокАзАТЕЛьство. Так как функционал под знаком интеграла Лебега в (7) неотрицателен на всей области интегрирования, то и сам интеграл неотрицателен. Поскольку, кроме того, $C>0$ в соответствии с (8), то $\mathscr{O}(y) \leqslant 1$. Далее, если $y(x)=\delta(x)$, то, как очевидно из $(7), \mathscr{O}(y)=1$. Покажем, что справедливо и обратное. Действительно, из свойств интеграла Лебега следует, что $\mathscr{O}(y)=1$ только в том случае, если функционалы (6) совпадают почти везде. Но так как эти функционалы являются обобщенными функциями, а следовательно, непрерьвны, они не могут совпадать почти везде, не совпадая везде на $\Phi$ в виду того, что мера $\mu$ обращается в нуль только на конечных или счетных множествах. Из последнего следует, что $\mathscr{O}(y)=1$ только в том случае, если $f_{1}(\varphi) \equiv f_{2}(\varphi)$, т.е. $y(x)=\delta(x)$.

ЛЕмма 3. Функиия $y(x)=\delta(x)$ является и при том единственной функиией, доставляющей относительный әкстремум функционалу $\mathscr{O}(y)$. Этот әкстремум максимум. 
ДокАЗАТЕЛЬСТво проведем в три этапа.

1) Необходимое условие экстремума. Вычислив вариацию от обеих частей равенства (7), получим

$$
\delta \mathscr{O}(y)=-2 C \int_{\Phi}\left(\int_{-a}^{a} y(x) \varphi(x) d x-\varphi(0)\right) \int_{-a}^{a} \varphi(\xi) \delta y(\xi) d \xi d \mu .
$$

При $y(x)=\delta(x)$ функционал под знаком интеграла Лебега тождественно равен нулю, в силу чего $\delta \mathscr{O}(\delta)=0$ для любой вариации $\delta y$.

2) Достаточное условие экстремума. Вычислив вторую вариацию, найдем

$$
\delta^{2} \mathscr{O}=-2 C \int_{\Phi}\left(\int_{-a}^{a} \varphi(x) \delta y(x) d x\right)^{2} d \mu .
$$

Так как функционал под знаком интеграла Лебега больше или равен нулю, причем может быть тождественно $(\forall \varphi \in \Phi)$ равен нулю, очевидно, только в случае, если $\delta y(x) \equiv$ 0, и так как $C>0$ (см. (3), (8)), то

$$
\delta^{2} \mathscr{O}<0
$$

при любых ненулевых вариациях $\delta y$. Следовательно, функция $y(x)=\delta(x)$ доставляет функционалу $\mathscr{O}(y)$ относительньй экстремум, причем - максимум.

3) Единственность экстремума. Покажем, что функция $y(x)=\delta(x)$ - единственная функция, удовлетворяющая необходимому условию экстремума.

Пусть для некоторой функции $y(x)$ имеет место равенство $\delta \mathscr{O}(y)=0 \quad \forall \delta y$. Вводя обозначение

$$
F(\varphi)=\int_{-a}^{a} y(x) \varphi(x) d x-\varphi(0)
$$

и меняя последовательность интегрирования в $(9)$ с учетом того, что $C \neq 0$, имеем

$$
\int_{-a}^{a} \delta y(x) d x \int_{\Phi} F(\varphi) \varphi(x) d \mu=0 \quad \forall \delta y(x) .
$$

В силу произвольности вариации это возможно, только если

$$
\int_{\Phi} F(\varphi) \varphi(x) d \mu \equiv 0
$$

Вычислим теперь $\rho^{2}(y, \delta)$. В соответствии с (5) и (10)

$$
\begin{aligned}
\rho^{2}(y, \delta) & =\int_{\Phi} F^{2}(\varphi) d \mu=\int_{\Phi} F(\varphi) d \mu \int_{-a}^{a}(y(x)-\delta(x)) \varphi(x) d x \\
& =\int_{-a}^{a}(y(x)-\delta(x)) d x \int_{\Phi} F(\varphi) \varphi(x) d \mu .
\end{aligned}
$$

Но в силу (11) внутренний интеграл в последнем выражении тождественно равен нулю, из чего следует, что $\rho^{2}(y, \delta)=0$, а значит, и $\rho(y, \delta)=0$. На основании аксиоматического определения метрики заключаем, что $y(x)=\delta(x)$. Итак, предполагая, что некоторая функция $y(x)$ удовлетворяет необходимому условию экстремума меры упорядоченности, мы пришли к выводу, что эта функция $-\delta$-функция. Таким образом, $\delta$-функция единственная функция, удовлетворяющая необходимому условию экстремума, а значит, единственная функция, доставляющая экстремум функционалу меры упорядоченности. 
4. Модельное уравнение. В начале работы мы привели примеры двух физических систем, переходящих из хаотического состояния в упорядоченное при изменении температуры в некотором интервале. Вообще говоря, параметр, управляющий самоорганизацией, - не обязательно температура (см. [1]). В общем случае будем рассматривать состояние статистической системы в зависимости от (управляющего) параметра $\tau: y=y(x, \tau)$. Пусть при $\tau=0$ система хаотична, т.е. описывается равномерным распределением:

$$
y(x, 0)=y_{0}=\frac{1}{2 a} .
$$

С ростом $\tau$ система упорядочивается. Можно предположить тот или иной закон упорядочения системы. Построим модель системы, которая развивается с ростом $\tau$ по закону наибы стрейшего роста порядка. Для этого потребуем, чтобы при любом $\tau$ изменение функции $y(x, \tau)$ в функциональном пространстве $\{y(x)\}$ происходило в направлении вектора градиента меры упорядоченности $\delta \mathscr{O} / \delta y$, т.е.

$$
\Delta y=y(x, \tau+\Delta \tau)-y(x, \tau)=\lambda(\tau) \frac{\delta \mathscr{O}}{\delta y} \Delta \tau+o(\Delta \tau)
$$

или

$$
\frac{\partial y}{\partial \tau}=\lambda(\tau) \frac{\delta \mathscr{O}}{\delta y}, \quad \lambda(\tau) \geqslant 0
$$

где $\lambda(\tau)$ - некоторая вещественная функция. Неотрицательность этой функции выражает требование роста (а не уменьшения) меры упорядоченности системы с ростом $\tau$. В дальнейшем мы будем также предполагать, что функция $\lambda(\tau)$ интегрируема на полуинтервале $[0,+\infty)$.

Запишем модельное уравнение (13) в развернутом виде. Подставляя (7) в (13), после несложных преобразований получим

$$
\frac{\partial y}{\partial \tau}=k \lambda(\tau) K(x, 0)-k \lambda(\tau) \int_{-a}^{a} K(x, \xi) y(\xi) d \xi
$$

где

$$
\begin{gathered}
k=2 C, \\
K\left(x_{1}, x_{2}\right)=\int_{\Phi} \varphi\left(x_{1}\right) \varphi\left(x_{2}\right) d \mu .
\end{gathered}
$$

Таким образом, модельное уравнение (13) представляет собой линейное неоднородное интегро-дифференциальное уравнение с интегральньм оператором типа Фредгольмa [11]:

$$
(\widehat{K} \psi)(x)=\int_{-a}^{a} K(x, \xi) \psi(\xi) d \xi .
$$

Отметим, что ядро этого оператора $K\left(x_{1}, x_{2}\right)$, так же как и мера упорядоченности $\mathscr{O}(y)$, определено неоднозначно, ибо является функцией меры множества $\Phi$ (см. (16)): $K\left(x_{1}, x_{2}\right)=K_{\mu}\left(x_{1}, x_{2}\right)$. Выберем меру $\mu$ так, чтобы ядро было непрерывно по обоим аргументам на сегменте $[-a, a]$ (предполагается, что последнее требование не противоречит аксиоматическому определению меры). 
В операторной форме записи уравнение (14) имеет вид

$$
\frac{\partial y}{\partial \tau}=k \lambda(\tau) \widehat{K}(\delta-y)
$$

Отметим, что поскольку $y(x)$ - это плотность вероятности, мы должны потребовать, чтобы решение начальной задачи $(18),(12)$ удовлетворяло условию нормировки

$$
\int_{-a}^{a} y(x, \tau) d x \equiv 1
$$

Этого можно добиться, предъявляя соответствующее требование к ядру $K\left(x_{1}, x_{2}\right)$.

ТЕорема 1. Пусть функиия $K\left(x_{1}, x_{2}\right)$ удовлетворяет условию

$$
\int_{-a}^{a} K\left(x_{1}, x_{2}\right) d x_{1} \equiv 1
$$

Тогда решение начальной задачи (18), (12) нормировано кединице.

ДокАЗАТЕЛЬСТво.Проинтегрировав по переменной $x$ обе части уравнения $(14)$, получим

$$
\frac{d}{d \tau} \int_{-a}^{a} y(x, \tau) d x=k \lambda(\tau) \int_{-a}^{a} K(x, 0) d x-k \lambda(\tau) \int_{-a}^{a}\left(\int_{-a}^{a} K(x, \xi) d x\right) y(\xi) d \xi
$$

В случае выполнения условия (20) это равенство принимает вид

$$
\frac{d}{d \tau} \int_{-a}^{a} y(x, \tau) d x=k \lambda(\tau)-k \lambda(\tau) \int_{-a}^{a} y(x, \tau) d x
$$

Вводя обозначение

$$
f(\tau)=\int_{-a}^{a} y(x, \tau) d x
$$

получим обыкновенное дифференциальное уравнение для функции $f(\tau)$ :

$$
\frac{d f}{d \tau}=k \lambda(\tau)(1-f)
$$

Его общее решение имеет вид

$$
f(\tau)=1-A e^{k \int_{0}^{\tau} \lambda(t) d t}, \quad A \in \mathbb{R}
$$

Из начального условия (12) видим, что $f(0)=1$. Соответствующее частное решение: $f(\tau) \equiv 1$. Таким образом, мы пришли к равенству (19).

В дальнейшем будем называть тождество (20) достаточным условием нормировки и предполагать его выполнение за счет соответствующего выбора меры $\mu$ в $(7),(16)$.

Отметим теперь основные свойства ядра $K\left(x_{1}, x_{2}\right)$. 
Лемма 4. Ядро симметрично: $K\left(x_{1}, x_{2}\right)=K\left(x_{2}, x_{1}\right)$.

Это свойство очевидно из определения (16).

Лемма 5. Ядро положительно определено [9]: $(\widehat{K} \psi, \psi) \geqslant 0, \psi \in L_{2}[-a, a]$.

ДокАЗАТЕльство. Подставляя выражение (16) в (17) и меняя порядок интегрирования, получим

$$
(\widehat{K} \psi)\left(x_{1}\right)=\int_{\Phi} \varphi\left(x_{1}\right) d \mu \int_{-a}^{a} \psi\left(x_{2}\right) \varphi\left(x_{2}\right) d x_{2} .
$$

Следовательно,

$$
(\widehat{K} \psi, \psi)=\int_{-a}^{a}(\widehat{K} \psi)\left(x_{1}\right) \psi\left(x_{1}\right) d x_{1}=\int_{-a}^{a} \psi\left(x_{1}\right) d x_{1} \int_{\Phi} \varphi\left(x_{1}\right) d \mu \int_{-a}^{a} \psi\left(x_{2}\right) \varphi\left(x_{2}\right) d x_{2} .
$$

После изменения порядка интегрирования в последнем выражении, имеем

$$
(\widehat{K} \psi, \psi)=\int_{\Phi}\left(\int_{-a}^{a} \psi(x) \varphi(x) d x\right)^{2} d \mu=\int_{\Phi}(\varphi, \psi)^{2} d \mu \geqslant 0
$$

что и требовалось доказать.

Лемма 6. Ядро финитно по обоим аргументам на $(-a, a)$.

ДокАЗАТЕЛЬСтво. Пусть, например, $x_{2}=c \notin(-a, a)$. Тогда

$$
K\left(x_{1}, x_{2}\right)=\int_{\Phi} F\left(x_{1}, c\right) d \mu
$$

где

$$
F\left(x_{1}, c\right)=\varphi\left(x_{1}\right) \varphi(c)
$$

при любом фиксированном $x_{1} \in \mathbb{R}$ - функционал от $\varphi$. Какой бы ни была функция $\varphi(x) \in$ $\Phi$, справедливо равенство $\varphi(c)=0$, так как $\Phi-$ множество основных функций на $[-a, a]$. Следовательно, функционал

$$
F\left(x_{1}, c\right) \equiv 0
$$

на всей области интегрирования $(\forall \varphi \in \Phi)$, а значит

$$
\int_{\Phi} F\left(x_{1}, c\right) d \mu=0
$$

(при любом фиксированном $x_{1} \in R$ ).

Итак, если $x_{2}=c \notin(-a, a)$, то $K\left(x_{1}, c\right) \equiv 0 \forall x_{1} \in \mathbb{R}$. Аналогично доказывается, что $K\left(x_{1}, x_{2}\right) \equiv 0$ при $x_{1} \notin(-a, a)$.

Лемма 7. Функиия $y_{0}$ является собственной функцией ядра, отвечающей собственному значению $\lambda=1$. 
ДокАзАТЕЛЬСтво. Действуя оператором Фредгольма (17) на функцию $y_{0}$, получим

$$
\widehat{K} y_{0}=\int_{-a}^{a} K(x, \xi) y_{0} d \xi=y_{0} \int_{-a}^{a} K(x, \xi) d \xi \equiv y_{0}
$$

в силу достаточного условия нормировки (20) и симметрии ядра. Таким образом,

$$
\widehat{K} y_{0}=1 \cdot y_{0}
$$

что и требовалось доказать. Отметим, что нормированной собственной функцией ядра будет не сама функция $y_{0}$, а функция $\sqrt{y_{0}} ;$ действительно,

$$
\int_{-a}^{a}\left(\sqrt{y_{0}}\right)^{2} d x=\int_{-a}^{a} y_{0} d x=1 .
$$

5. Решение модельного уравнения. Найдем решение модельного уравнения (18), удовлетворяющее начальному условию (12). Для этого введем замену переменной $\tau \rightarrow$ $t$, определенную соотношениями

$$
\lambda(\tau)=\frac{d t(\tau)}{d \tau}, \quad t(0)=0
$$

т.е.

$$
t=\int_{0}^{\tau} \lambda(\theta) d \theta
$$

В результате начальная задача (18), (12) преобразуется к виду

$$
\begin{gathered}
\frac{\partial y}{\partial t}=k K(x, 0)-k \widehat{K} y \\
y(x, 0)=y_{0}
\end{gathered}
$$

Будем искать решение этой задачи в форме разложения по собственньм функциям $y_{n}(x)$ оператора Фредгольма $\widehat{K}[11]$, определенньгм равенством:

$$
\widehat{K} y_{n}=\lambda_{n} y_{n}, \quad n=1,2, \ldots
$$

где $\lambda_{n}$ - собственные значения. При этом предположим следующее.

1) Оператор $\widehat{K}$ не имеет нулевых собственных значений (т.е. ядро $K\left(x_{1}, x_{2}\right)$ невырождено): $\lambda_{n} \neq 0 ; n=1,2, \ldots$.

2) Ранг каждого собственного значения равен единице.

3) Для определенности положим, что собственное значение $\lambda=1$, отвечающее равномерному распределению $y_{0}$, является первым в последовательности $\left\{\lambda_{n}\right\}: \lambda_{1}=1$.

В виду того, что свободный член интегро-дифференциального уравнения (23) на основании теоремы Мерсера [11] разложим по базису из собственных векторов оператора $\widehat{K}$ :

$$
K(x, 0)=\sum_{n=1}^{\infty} \lambda_{n} y_{n}(0) y_{n}(x)
$$


а начальная функция $y(x, 0)=y_{0}$ сама является собственным вектором ядра, будем сразу искать решение задачи $(23),(24)$ в виде

$$
y(x, t)=\sum_{n=1}^{\infty} u_{n}(t) y_{n}(x) .
$$

Подставляя это выражение в уравнение (23), используя (26) и приравнивая коэффициенты при ортогональных функциях $y_{n}(x)$, получим обькновенное дифференциальное уравнение для $u_{n}(t)$ :

$$
\dot{u}_{n}+k \lambda_{n} u_{n}=k \lambda_{n} y_{n}(0), \quad n=1,2, \ldots
$$

Общее решение этого уравнения имеет вид

$$
u_{n}(t)=y_{n}(0)+A_{n} e^{-k \lambda_{n} t}, \quad A_{n} \in \mathbb{R}
$$

Начальное условие (24) с учетом разложения (27) и линейной независимости собственных функций $y_{n}(x)$ дает

$$
u_{1}(0)=\sqrt{y_{0}}, \quad u_{n}(0)=0, \quad n=2,3, \ldots
$$

откуда

$$
A_{1}=0, \quad A_{n}=-y_{n}(0), \quad n=2,3, \ldots .
$$

Таким образом, для $u_{n}(t)$ имеем

$$
\begin{gathered}
u_{1}(t)=y_{1}(0)=\sqrt{y_{0}} \\
u_{n}(t)=y_{n}(0)-y_{n}(0) e^{-k \lambda_{n} t}, \quad n=2,3, \ldots
\end{gathered}
$$

Подставляя эти выражения в (27), получим

$$
y(x, t)=y_{0}+\sum_{n=2}^{\infty}\left(1-e^{-k \lambda_{n} t}\right) y_{n}(0) y_{n}(x) .
$$

Это и есть искомое решение задачи (23), (24).

Убедимся, что ряд в (29) сходится абсолютно и равномерно. Для этого покажем, что коэффициенты этого ряда удовлетворяют оценке

$$
1-e^{-k \lambda_{n} t}<k \lambda_{n} t .
$$

Рассмотрим интеграл

$$
I=\int_{0}^{t} e^{-k \lambda_{n} \theta} d \theta
$$

Taк как

$$
0<e^{-k \lambda_{n} \theta}<1, \quad n=2,3, \ldots, \quad \theta>0,
$$

TO

$$
I<\int_{0}^{t} d \theta=t
$$


С другой стороны,

$$
I=\frac{1}{k \lambda_{n}}\left(1-e^{-k \lambda_{n} t}\right) .
$$

Сравнивая (31) и (32), приходим к оценке (30). Из нее следует оценка для общего члена ряда в (29):

$$
\left|\left(1-e^{-k \lambda_{n} t}\right) y_{n}(0) y_{n}(x)\right|<k \lambda_{n} t\left|y_{n}(0)\right|\left|y_{n}(x)\right|
$$

Однако справа в этом неравенстве стоит домноженньй на $k t$ модуль общего члена ряда $(26)$ для $K(x, 0)$. В силу того, что ряд $(26)$ сходится абсолютно и равномерно (по теореме Мерсера), ряд в (29) также сходится абсолютно и равномерно. Равномерная сходимость ряда в (29) позволяет его почленно интегрировать.

Для обоснования корректности почленного дифференцирования ряда в (29), докажем равномерную сходимость ряда

$$
\sum_{n=2}^{\infty}\left(1-e^{-k \lambda_{n} t}\right)^{\prime} y_{n}(0) y_{n}(x)
$$

Этот ряд, очевидно, равен

$$
k \sum_{n=2}^{\infty} e^{-k \lambda_{n} t} \lambda_{n} y_{n}(0) y_{n}(x)
$$

и так как

$$
e^{-k \lambda_{n} t} \leqslant 1 \quad \text { при } t \in[0, \infty),
$$

он сходится абсолютно и равномерно по тем же причинам, что и ряд в (29).

Непосредственной подстановкой можно проверить, что функция (29) действительно является решением уравнения (23). Очевидно также, что это решение удовлетворяет и начальному условию (24). Покажем, что оно нормировано. На основании теоремы Мерсера положительно определенное, симметричное ядро $K(x, \xi)$ представимо в виде

$$
K(x, \xi)=y_{0}+\sum_{n=2}^{\infty} \lambda_{n} y_{n}(x) y_{n}(\xi)
$$

Интегрируя обе части этого равенства по переменной $\xi$ и используя условие (20), получим

$$
\int_{-a}^{a} K(x, \xi) d \xi=1+\sum_{n=2}^{\infty} \lambda_{n} y_{n}(x) \int_{-a}^{a} y_{n}(\xi) d \xi \equiv 1 .
$$

В виду линейной независимости собственных функций $y_{n}(x)$ из последнего следует, что

$$
\int_{-a}^{a} y_{n}(\xi) d \xi=0, \quad n=2,3, \ldots
$$

Интегрируя теперь решение (29) по $x$ видим, что в силу последнего равенства оно нормировано к единище.

Отметим важное свойство решения (29). 
Теорема 2. Решение (29) задачи (23), (24) стремится (в смысле слабой сходимости) $\kappa \delta$-функции при $t \rightarrow+\infty:$

$$
y(x, t) \rightarrow \delta(x), \quad t \rightarrow+\infty .
$$

ДоКАЗАТЕЛЬСТВо. В виду положительной определенности ядра $K\left(x_{1}, x_{2}\right)$ все его собственные значения неотрицательны [9]:

$$
\lambda_{n} \geqslant 0, \quad n=1,2, \ldots .
$$

Поскольку же ядро не вырождено, т.е. не имеет нулевого собственного значения, и поскольку $k>0$, то для всех членов суммы в (29) вьполнено

$$
e^{-k \lambda_{n} t} \rightarrow 0, \quad t \rightarrow+\infty
$$

Следовательно,

$$
y(x, t) \rightarrow y_{0}+\sum_{n=2}^{\infty} y_{n}(0) y_{n}(x), \quad t \rightarrow+\infty
$$

Ho

$$
y_{0}+\sum_{n=2}^{\infty} y_{n}(0) y_{n}(x)=\sum_{n=1}^{\infty} y_{n}(0) y_{n}(x),
$$

а последний ряд есть ни что иное, как разложение $\delta$-функции по собственньм векторам оператора Фредгольма [12]:

$$
\sum_{n=1}^{\infty} y_{n}(0) y_{n}(x)=\delta(x)
$$

откуда непосредственно следует соотношение (33).

Суть доказанной теоремы состоит в том, что система, находящаяся при $t=0$ в хаотическом состоянии $y_{0}$ и развивающаяся с ростом $t$ по закону наибыстрейшего роста порядка, в пределе при $t \rightarrow+\infty$ достигает наиболее упорядоченного состояния.

Решение задачи (18), (12) получим, подставляя в (29) выражение (22) для $t$ :

$$
y(x, \tau)=y_{0}+\sum_{n=2}^{\infty}\left(1-e^{-k \lambda_{n} \int_{0}^{\tau} \lambda(\theta) d \theta}\right) y_{n}(0) y_{n}(x) .
$$

Если функция $\lambda(\tau)$ определена так, что

$$
t(\tau) \rightarrow+\infty
$$

при некотором стремлении $\tau$ :

$$
\tau \rightarrow \tau_{m} \quad \text { или } \quad \tau \rightarrow+\infty,
$$

то в силу теоремы 2 решение (34) будет стремиться к $\delta$-функции при этом стремлении $\tau$ :

$$
y(x, \tau) \rightarrow \delta(x),
$$

и система будет достигать наиболее упорядоченного состояния в соответствующем пределе. Наоборот, если соотношение (35) не имеет места ни при каком стремлении $\tau$, то наиболее упорядоченное состояние, по-видимому, недостижимо.

В заключение отметим, что поскольку $y(x, \tau)$ - плотность вероятности, следует потребовать неотрицательности этой функции. Возможно, это требование приведет к дополнительным условиям на ядро $K\left(x_{1}, x_{2}\right)$ и, тем самым, на меру $\mu$. 


\section{СПИСОК ЦИТИРОВАННОЙ ЛИТЕРАТУРЫ}

[1] Хакен Г. Синергетика. М.: Мир, 1980.

[2] Пригожин И., Стенгерс И. Порядок из хаоса. М.: Прогресс, 1986.

[3] Климонтович Ю. Л. Турбулентное движение и структура хаоса. М.: Наука, 1990.

[4] Ма Ш. Современная теория критических явлений. М.: Мир, 1980.

[5] Пелюхова Е. Б., Фрадкин Э.Е. Самоорганизация физических систем. С.-Пб.: Изд-во С.-Петербургского ун-та, 1997.

[6] Климонтович Ю. Л. Энтропия и информация открытых систем // УФН. 1999. Т. 169. C. 443.

[7] Сергеев Э. В., Тремаскин А. В. Синергетика вибрационных квази-циклических колебаний // Необратимые процессы в природе и технике. М.: МГТУ им. Н. Э. Баумана, 2001.

[8] Киттель Ч. Введение в физику твердого тела. М.: Наука, 1978.

[9] Владимиров В. С. Уравнения математической физики. М.: Наука, 1971.

[10] Колмогоров А. Н., Фомин С. В. Элементы теории функций и функционального анализа. М.: Наука, 1976.

[11] Васильева А. Б., Тихонов Н. А. Интегральные уравнения. М.: МГУ, 1989.

[12] Тихонов А. Н., Самарский А. А. Уравнения математической физики. М.: Наука, 1966.

Московский государственный технический университет

Поступило

им. Н. Э. Баумана

17.05 .2002

Исправленный вариант

30.01 .2003 